\title{
Setaria pujoli,
}

\section{nouvelle Filaire parasite d'une Antilope : Neotragus batesi De Winton, originaire du Cameroun}

\author{
par Marie-Claude DURETTE-DESSET
}

Laboratoire de Zoologie (Vers), associé au C.N.R.S. (Pr A.-G. Chabaud), Muséum national d'Histoire naturelle, 57, rue Cuvier, F 75005 Paris

\section{Résumé}

Description de Setaria pujoli n. sp., parasite de Neotragus batesi au Cameroun. Cette Sétaire, proche par ses structures céphaliques de 4 Sétaires parasites de Cephalophinae, s'en éloigne par la disposition particulière des papilles cloacales chez le mâle.

\section{Summary}

Setaria pujoli n. sp., parasite of Neotragus batesi from Cameroon is described. The cephalic structures of this Filaria are related to those of four species, parasites of Cephalophinae, but the new species is easily recognized by the peculiar distribution of the post-cloacal papillae.

A notre connaissance, aucun Nématode n'a encore été décrit chez les Antilopes du genre Neotragus: $N$. pigmaeus L. et $N$. batesi De Winton.

L'envoi de deux lots de Nématodes (*), provenant de la cavité péritonéale de deux $N$. batesi, nous a permis la description d'une nouvelle espèce de Filaire du genre Setaria.

(*) Nous remercions vivement le $\mathrm{D}^{\mathrm{r}} \mathrm{A}$. Pujol qui nous a rapporté des spécimens de Yaoundé et le $\mathrm{D}^{\mathrm{r}} \mathrm{P}$. M. Troncy qui nous a transmis des spécimens récoltés par le $\mathrm{D}^{\mathrm{r}}$ Garcin que nous remercions également. 
Les espèces les plus proches sont parasites de Cephalophinae qui vivent en forêt. C'est également le cas du Neotragus batesi qui appartient à une sous-famille (Raphicerinae) placée du point de vue systématique juste avant les Cephalophinae (cf. Grassé, 1955).

Les spécimens étudiés sont déposés dans les collections du Muséum national d'Histoire naturelle de Paris.

\section{Description de Setaria pujoli n. sp.}

- Matériel type : $2 \delta^{\star}, 15$, récoltés chez un Neotragus batesi De Winton, originaire du village d'Eyang à $15 \mathrm{~km}$ de Yaoundé (Cameroun) — tube $735 \mathrm{Ka}$.

- Autre matériel : 4 q récoltées chez le même hôte et dans la même région tube $113 \mathrm{Ka}$.

\section{Deseription :}

Corps blanc, cylindrique, aminci à l'extrémité postérieure légèrement enroulée chez la femelle, plus fortement chez le mâle.

Ouverture buccale ronde, de $15 \mu$ de diamètre, entourée d'un anneau chitinoïde ovalaire long de $37 \mu$ (axe dorso-ventral) et large de $28 \mu$ (axe latéral) (fig. 1, B).

Les dents médianes sont hautes de $12 \mu$ et assez rapprochées l'une de l'autre; leur bord interne se raccorde avec le pourtour buccal selon une pente assez douce. La distance entre les dents est de $28 \mu$ au niveau des sommets et de $40 \mu$ à la base. En vue médiane, les sommets sont arrondis (fig. 1, D).

Les deirides sont symétriques ou légèrement asymétriques, situées un peu en avant de la fin de l'œsophage musculaire (fig. 1, A). Elles possèdent une pointe unique, arrondie (fig. 1, G, H). Nous avons observé que la base de la deiride est plus ou moins allongée selon l'épaisseur de la cuticule. Quand la femelle est jeune, la cuticule est moins épaisse et la base de la deiride plus courte (fig. 1, J).

MÂle : Corps long de $4 \mathrm{~cm}$ sur $300 \mu$ de large dans sa partie moyenne. Anneau nerveux et deirides situés respectivement à $190 \mu$ et $400 \mu$ de l'apex. Esophage long de $3150 \mu$ (musculaire : $450 \mu$, glandulaire : $2700 \mu$ ).

L'extrémité postérieure du corps est enroulée sur elle-même sur une longueur de $4 \mathrm{~mm}$. Les annelures de l'area rugosa débutent à $3 \mathrm{~mm}$ de l'extrémité caudale et se terminent entre la première et la deuxième paire de papilles précloacales. Dans la partie proximale de l'area rugosa, les annelures sont espacées de $22 \mu$ (fig. 2, E) ; cette distance diminue progressivement et n'est plus que de $9 \mu$ dans la partie distale. 

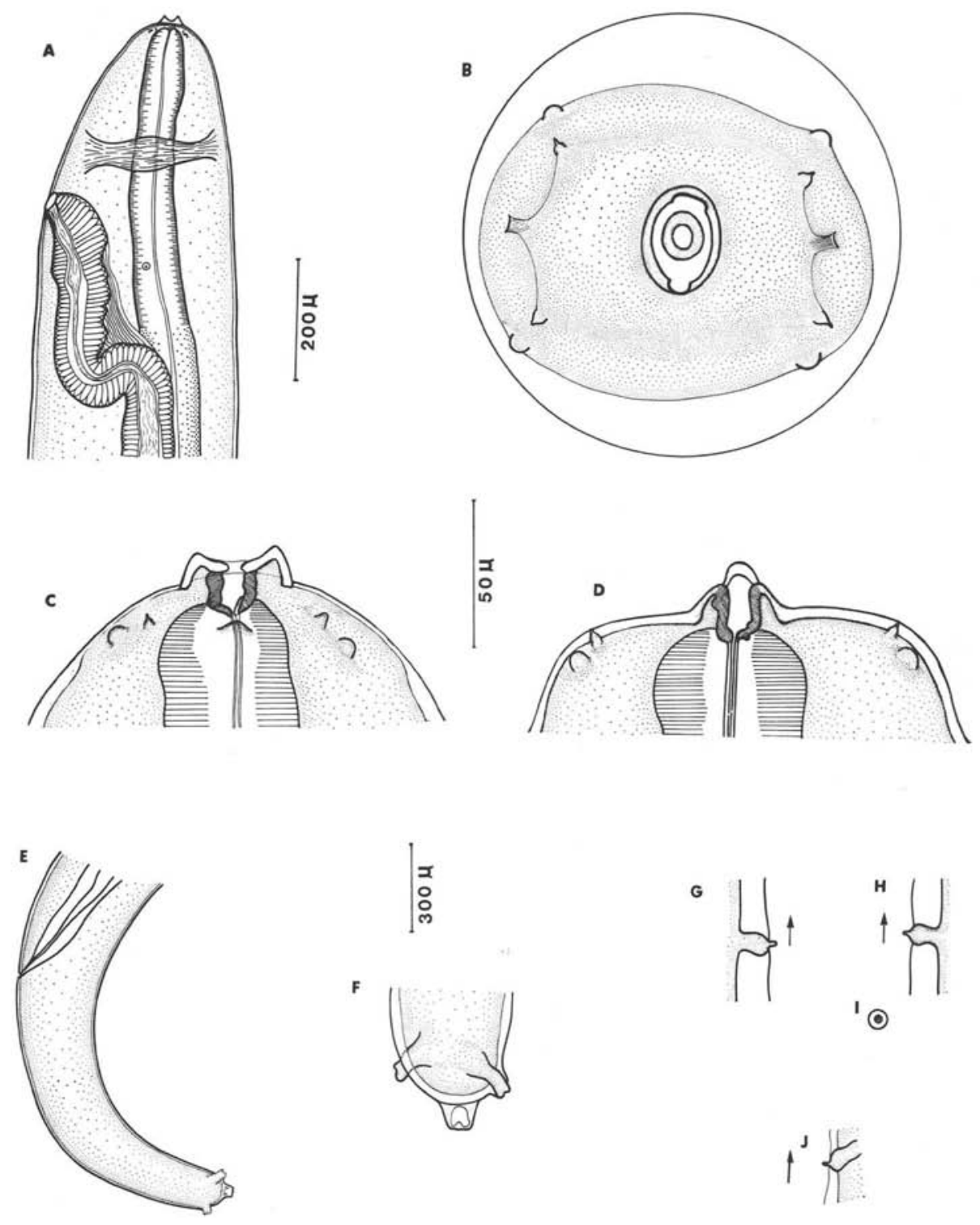

1

FIg. 1. - Setaria pujoli n. sp. ‥ A. - Extrémité antérieure, vue latérale gauche. B. - Tête, vue apicale. C. - id., vue latérale. D. - id., vue médiane. E. - Extrémité postérieure, vue latérale gauche. F. - Pointe caudale, vue latérale gauche. G., H. - Deirides gauche et droite, vue latérale.

I. - Deiride droite, vue de face. J. - Autre spécimen, $q$, deiride droite, vue latérale.

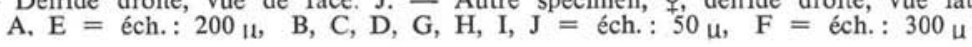


Queue large de $175 \mu$, portant 9 papilles précloacales dont une papille impaire et 7 paires de papilles post-cloacales plus une paire de phasmides. La disposition de ces papilles est caractéristique, les 2 premières paires de papilles post-cloacales étant nettement séparées de toutes les autres qui sont situées à l'extrémité de la queue (fig. 2, B).

Le petit spicule long de $115 \mu$ et large de $30 \mu$ a la forme générale d'une gouttière, mais il est de structure complexe. La partie proximale de la face latérale droite se prolonge en avant par une plaque épaisse; la pointe membraneuse distale est déportée vers la droite (fig. 2, F).

Le grand spicule est composé de deux parties: le manche chitinoïde est long de $300 \mu$ sur $30 \mu$ de large, la lame membraneuse est longue de $430 \mu$. Elle s'insère sur le manche sur une hauteur de $130 \mu$ (fig. 2, D).

Femelle: Corps long de $13 \mathrm{~cm}$ sur $800 \mu$ de large dans sa partie moyenne. Anneau nerveux et deirides situés respectivement à $210 \mu$ et $420 \mu$ de l'apex. Esophage long de $3910 \mu$ (musculaire : $510 \mu$, glandulaire : $3400 \mu$ ).

La vulve s'ouvre juste en arrière de l'anneau nerveux à $330 \mu$ de l'apex. Le sphincter, allongé, s'étend jusqu'à $370 \mu$ en arrière de la vulve (fig. 1, A). La séparation en deux utérus a lieu à $1,4 \mathrm{~mm}$ de l'apex.

Queue longue de $610 \mu$ portant à son extrémité un bouton caudal arrondi plus ou moins lobé selon les spécimens (fig. 1, E). Deux pétales caudaux, presque aussi gros que le bouton terminal, et généralement bifides, sont situés à $40 \mu$ de l'extrémité caudale (fig. 1, F).

Les œufs mesurent $33 \mu \times 18 \mu$. Les microfilaires sont longues de $310 \mu$ et larges de $10 \mu$.

\section{Discussion :}

Les caractères céphaliques (disposition et forme des dents) rapprochent ces spécimens de quatre espèces africaines. Toutes les quatre sont parasites de Cephalophinae: $S$. dipetalonematoides Chabaud et Rousselot, 1956, au Congo, S. gaillardi Desset, 1966, au Sénégal, $S$. machadoi Desset, 1966, au Congo et $S$. southwelli Twaite, 1927, en Sierra Leone.

Cependant, les spécimens du Neotragus se différencient aisément de ces quatre espèces par la disposition particulière des papilles post-cloacales chez le mâle (un groupe est situé juste en arrière de l'anus, l'autre à l'extrémité caudale). Par contre, une telle disposition se retrouve chez $S$. kabargi Kadenazii, 1948, parasite de Moscus moschiferus en Russie et S. cervicapra Shoho, 1966, parasite d'Antilope capra en Indes, mais les autres caractères de ces deux espèces et, en particulier, la structure céphalique n'ont pas d'affinités avec la nôtre.

Nous pensons donc que nos spécimens sont nouveaux et nous proposons le nom de Setaria pujoli n. sp. 


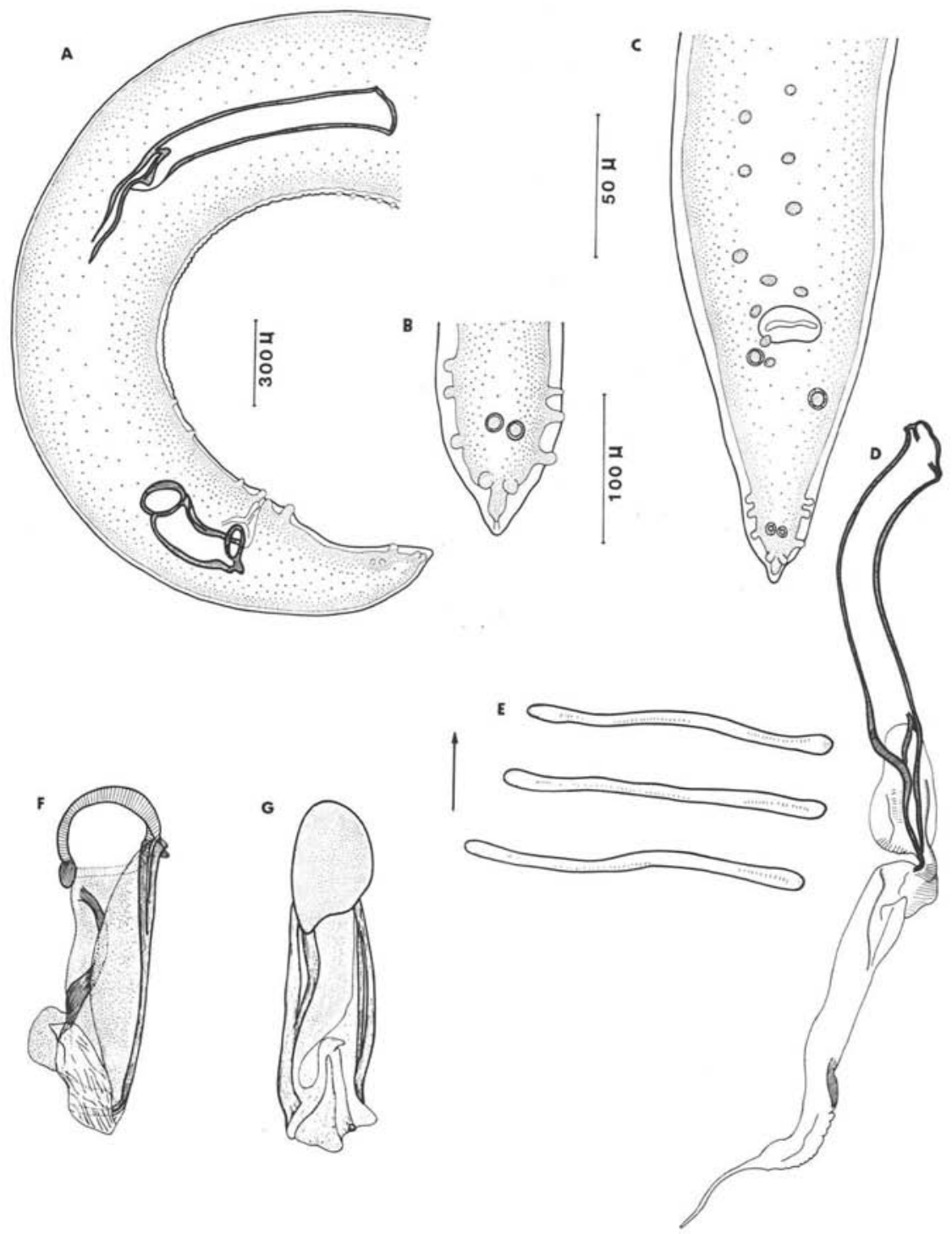

Fig. 2. - Setaria pujoli n. sp. §. A. - Extrémité postérieure, vue latérale droite. B. - pointe caudale, vue ventrale. C. - Extrémité postérieure, vue ventrale D. - Grand spicule disséqué. E. - Détail de l'area rugosa. F. - Petit spicule, vue sub.-ventrale gauche. G. - id. vue ventrale $\mathrm{A}=$ éch. : $300_{\mu}, \mathrm{B}, \mathrm{D}, \mathrm{E}, \mathrm{F}, \mathrm{G}=$ éch. : $100_{\mu}, \mathrm{C}=$ éch. $50_{\mu}$ 
Il est intéressant de remarquer que la sous-famille des Raphicerinae à laquelle appartiennent les Neotragus est proche de celle des Cephalophinae à laquelle appartiennent les quatre espèces les plus proches en ce qui concerne les caractères cépháliques. On peut aussi noter que tous ces Bovidae sont des animaux de forêt.

\section{Bibliographie}

Chabaud (A.-G.) et Rousselot (R.), 1956. - Sur quelques Filaires d'Afrique Equatoriale. Ann. Parasit. hum. comp., 30, 53-98.

DeSSET (M.-C.). 1966. - Contribution à la systématique des Filaires du genre Setaria; valeur des deirides. Mém. Mus. Nat. Hist. Nat., n. sér. Sér. A, Zool., 39, 257-286.

Grasse (P.-P.), 1955. - Traité de Zoologie. Tome 17. Premier fascicule. Masson et $C^{i e}$ éd., Paris.

Kadenazi in SkrJabin (K. I.) et Shikhobalova (N. P.), 1948. - «Filyarii zhivotnykh i cheloveka », Moscow : Ogiz - Sel'korgiz.

Sноно (C.), 1966. - Description de Setaria cervicapra n. sp. parasite d'Antilope cervicapra en Inde et note sur la structure cuticulaire de S. capreola. Ann. Parasit. hum. comp., 41, 487-493.

Twaite (J.W.), 1927. - The genus Setaria. Ann. trop Med. Parasit., 21, 427-466. 